\title{
Phenotypic and genotypic characterization of two closely related subgroups of Candida rugosa in clinical specimens
}

Correspondence

Sun Tee Tay

tayst@um.edu.my

Received 2 April 2011

Accepted 21 June 2011

\author{
Sun Tee Tay, Hui Wee Tan, Shiang Ling $\mathrm{Na}$ and Su Lin Lim \\ Department of Medical Microbiology, Faculty of Medicine, University of Malaya, 50603 Kuala \\ Lumpur, Malaysia
}

\section{INTRODUCTION}

Candida rugosa has been recognized as an emerging fungal pathogen capable of causing invasive infection in immunocompromised patients. This yeast has been identified as a rare cause of bloodstream infections in North America and Europe (Minces et al., 2009) and accounted for $0.4 \%$ of Candida isolates in the global ARTEMIS DISK Antifungal Surveillance Program (Pfaller et al., 2006). However, it has been noted with increased frequency of isolation in several geographical regions recently. C. rugosa has been identified as a frequent colonizer of high-risk patients and accounted for $44 \%$ of 32 consecutive episodes of candidaemia in critically ill Brazilian patients (Colombo et al., 2003; Rosas et al., 2004). In a laboratory-based

Abbreviation: ITS, intergenic transcribed spacer.

The GenBank/EMBL/DDBJ accession numbers for the C. rugosa sequences determined in this study are HQ412589 and HQ412590 (D1/D2 domain) and HM641831 and HM641832 (ITS sequence) for subgroups $A$ and $B$, respectively. surveillance study in India, $C$. rugosa was the predominant Candida species isolated from the blood cultures of $>20 \%$ of critically ill trauma patients (Behera et al., 2010; Singh et al., 2011).

The existence of genetic heterogeneity in C. rugosa isolates has been documented in a previous study where clinical isolates were clustered into nine fingerprinting groups using a repetitive sequence-based PCR technique (Redkar et al., 1996). Recently, a novel yeast species closely related to $C$. rugosa, Candida pseudorugosa, exhibiting a similar API 20C AUX carbohydrate assimilation profile to C. rugosa, has been reported (Li et al., 2006). Reports on the association of C. rugosa with catheters and total parenteral nutrition, nosocomial spread and decreased susceptibility to both polyenes and fluconazole (Dubé et al., 1994; Minces et al., 2009) have made identification and characterization of this emerging yeast pathogen important.

Molecular methods based on sequencing of the 26S rRNA gene D1/D2 domain or intergenic transcribed spacer (ITS) 
region and use of carbohydrate assimilation profiling and chromogenic Candida agar are helpful for the correct identification of clinical isolates of yeast when routine phenotypic methods are insufficient (Ciardo et al., 2006; Kurtzman \& Robnett, 1997). To validate the identity of our isolates, phenotypic and molecular approaches including enzymic analysis were applied. The antifungal susceptibilities of the planktonic and sessile cells of these isolates are also presented in this study.

\section{METHODS}

Yeast isolates. Table 1 shows the origin and details of six yeast isolates (strains STC1-6), which were initially identified as C. rugosa by API 20C AUX tests (code 6442104; bioMérieux) in this study. The yeasts were obtained from blood $(n=2)$, urine $(n=2)$, a vaginal swab $(n=1)$ and a bronchoalveolar lavage $(n=1)$ from six independent inpatients at the University of Malaya Medical Center, Kuala Lumpur, Malaysia, from 2005 to 2007. The yeasts were inoculated onto potato dextrose agar and incubated at $25,30,35,42$ and $45{ }^{\circ} \mathrm{C}$ for up to 7 days. Chromogenic testing of the isolates was performed on Oxoid Chromogenic Candida Agar (OCCA). An ID32 C kit (bioMérieux) was used for further identification of the yeast strains, following the manufacturer's instructions.

Enzymic profiling of C. rugosa. API ZYM (bioMérieux) analysis was used to define the enzymic profiles of the yeasts, as recommended by the manufacturer. A proteinase agar clearance assay was performed according to the method of Ruma-Haynes et al. (2000).

Sequence determination and analysis. Yeast genomic DNA was extracted as described by Makimura et al. (1994). The ITS region and the 26S rRNA gene D1/D2 domain were amplified with primer pair ITS1 (5'-GTCGTAACAAGGTTTCCGTAGGTG-3') and ITS4 (5'TCCTCCGCTTATTGATATGC-3') (White et al., 1990) and primer pair NL1 (5'-GCATATCAATAAGCGGAGGAAAAG-3') and NL4 (5'-GGTCCGTGTTTCAAGACGG-3'), respectively (Kurtzman \& Robnett, 1997). The amplicon was purified using a PCR SV kit (GeneAll) and the subsequent sequencing reaction was performed with a Big Dye Terminator version 3.1 Cycle Sequencing kit (Applied Biosystems) on an ABI PRISM 377 Genetic Analyzer (Applied Biosystems), using forward and reverse primers. The sequences were used for a BLAST search in GenBank. The neighbour-joining method in MEGA version 4.0 was used to determine the phylogenetic status of the isolates (Kumar et al., 2004). The reliability of different phylogenetic groupings was evaluated using bootstrap tests (1000 bootstrap replications). Sequences of C. rugosa isolates (Table 2) and reference strains of Candida species were retrieved from GenBank.

Determination of planktonic MICs. The planktonic MICs of amphotericin, fluconazole, ketoconazole and flucytosine for the yeasts were determined using a microbroth dilution method as described by the Clinical and Laboratory Standards Institute (CLSI, 2008). MICs were recorded after incubation at $35{ }^{\circ} \mathrm{C}$ for $48 \mathrm{~h}$. The MIC of amphotericin was defined as the lowest concentration at which there was an absence of growth. The MICs of the other drugs were defined as the lowest drug concentrations that achieved $80 \%$ growth inhibition compared with growth of the drug-free control.

Determination of biofilm formation and MICs. The biofilmforming abilities of the isolates were determined using a standardized method as described by Ramage et al. (2001). Briefly, an overnight yeast culture was suspended in RPMI 1640 broth supplemented with L-glutamine (Sigma) to a final concentration of $10^{7}$ cells $\mathrm{ml}^{-1}$

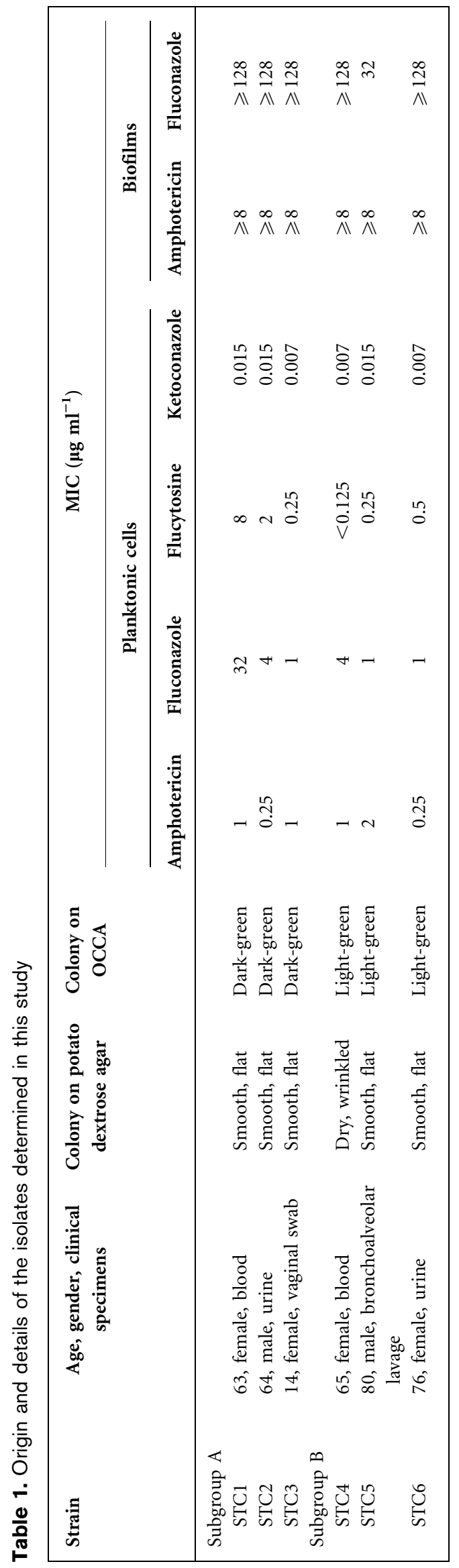


Table 2. Origin and details of isolates of the C. rugosa complex and its closely related species used for phylogenetic analysis in this study

\begin{tabular}{|c|c|c|c|}
\hline Strains & Source & GenBank no. (gene) & Reference \\
\hline $\begin{array}{l}\text { Candida rugosa strain } \\
\text { ATCC } 10571\end{array}$ & ATCC strain & GU144663 (D1/D2 domain/ITS) & $\begin{array}{l}\text { A. J. Mota and others } \\
\text { (unpublished) }\end{array}$ \\
\hline Candida sp. L2683B & Blood, Brazil & $\begin{array}{l}\text { FJ768918 (D1/D2 domain), } \\
\text { FJ768913 (ITS) }\end{array}$ & $\begin{array}{l}\text { G. M. Chaves and others } \\
\text { (unpublished) }\end{array}$ \\
\hline Candida rugosa $\mathrm{UOA} / \mathrm{HCPF} 2$ & Blood, Australia & GQ376074 (ITS) & $\begin{array}{l}\text { W. Meyer and others } \\
\text { (unpublished) }\end{array}$ \\
\hline Candida rugosa LYSM3 & Soil from forest, Thailand & AB498988 (D1/D2 domain) & $\begin{array}{l}\text { R. Kaewwichian and others } \\
\text { (unpublished) }\end{array}$ \\
\hline Candida rugosa $\mathrm{EB} 2$ & $\begin{array}{l}\text { Water from mangrove forest, } \\
\text { Thailand }\end{array}$ & AB436404 (D1/D2 domain) & $\begin{array}{l}\text { C. Boonmak and others } \\
\text { (unpublished) }\end{array}$ \\
\hline Candida rugosa Zhuan8 & Marine yeast, China & EF197805 (ITS) & N. Guo and Z. Chi (unpublished) \\
\hline Subgroup A (STC1) & Clinical specimen, Malaysia & $\begin{array}{l}\text { HQ412589 (D1/D2 domain), } \\
\text { HM641831 (ITS) }\end{array}$ & This study \\
\hline Subgroup B (STC4) & Clinical specimen, Malaysia & $\begin{array}{l}\text { HQ412590 (D1/D2 domain), } \\
\text { HM641832 (ITS) }\end{array}$ & This study \\
\hline
\end{tabular}

$\left(\mathrm{OD}_{530}\right.$ of 0.38$)$. An aliquot of $100 \mu \mathrm{l}$ of the yeast suspension was then transferred to each well of a flat-bottomed, 96-well polystyrene microtitre plate (Nunclon). After incubation with shaking at 75 r.p.m. at $37{ }^{\circ} \mathrm{C}$ for $1.5 \mathrm{~h}$, unattached cells were removed by washing twice with $150 \mu \mathrm{l}$ PBS. Fresh RPMI $1640(100 \mu \mathrm{l})$ was then added to each well to allow proliferation of the biofilm for $24 \mathrm{~h}$. The metabolic activity of the biofilm was measured using a 2,3-bis(2methoxy-4-nitro-5-sulfophenyl)-5-[(phenylamino)carbonyl]-2H-tetrazolium hydroxide (XTT) reduction assay as described by jin et al. (2003). The biofilm in each well was washed twice with $200 \mu \mathrm{l} \mathrm{PBS}$ and a solution containing XTT with menadione was added. The $A_{490}$ of the resultant solution $(100 \mu \mathrm{l})$ after incubation for $1 \mathrm{~h}$ in the dark was measured with a spectrophotometer. The biofilm MIC was determined as described by Melo et al. (2007). Biofilms were washed after $24 \mathrm{~h}$, and amphotericin and fluconazole were added at concentrations of $0.015-8$ and $0.25-128 \mu \mathrm{g} \mathrm{ml}^{-1}$, respectively. Two hundred microlitres of each drug solution was added to the respective wells of the microtitre plate and incubated at $37{ }^{\circ} \mathrm{C}$ for $48 \mathrm{~h}$. Biofilm MICs were determined on the basis of a $50 \%$ reduction in metabolic activity compared with the activity of the biofilm in the microtitre well without the drug. Experiments were performed in duplicate for each isolate.

\section{RESULTS}

\section{Phenotypic characterization}

Five isolates produced morphologically similar flat, butyrous and creamy colonies on potato dextrose agar, whilst one isolate (strain STC4, subgroup B) grew dry and wrinkled colonies. Identification using an ID32 C test kit revealed similar carbohydrate assimilation profiles (code 1300310015) for the six isolates and identified the isolates as C. rugosa. The isolates were differentiated into two subgroups based on their pigmentation on OCCA. Strains STC1-3 (designated subgroup A) developed darker green colonies compared with the rest of the isolates (designated subgroup B). All isolates were able to grow at 25, 30, 35 and $42{ }^{\circ} \mathrm{C}$ but not at $45{ }^{\circ} \mathrm{C}$.

\section{Enzymic characterization}

Identical enzymic profiles were obtained by API ZYM analysis for a representative isolate from each subgroup in this study, with the detection of alkaline phosphatase, esterase, leucine arylamidase, acid phosphatase, esterase lipase and lipase (C14) from the isolates. Proteinase production was detected from all isolates on proteinase clearance agar after 7 days of incubation.

\section{Sequence analysis of the D1/D2 domain}

A total of 466 and $467 \mathrm{nt}$ were determined for the D1/D2 domain of subgroup A isolates (accession no. HQ412589) and subgroup B isolates (accession no. HQ412590), respectively. There was no mismatch in the $\mathrm{D} 1 / \mathrm{D} 2$ domain within the subgroups. Table 3 shows a summary of the sequence analysis. Other than the difference in sequence length, the two subgroups differed by three mismatches. Subgroup A isolates demonstrated $99.1 \%$ (four mismatches) nucleotide similarity with the C. rugosa ATCC 10571 type strain, whilst subgroup B isolates demonstrated $99.6 \%$ (two mismatches including an insertion) nucleotide 
Table 3. Comparison of the D1/D2 domain and ITS gene regions of the isolates in this study with those of reference strains

\begin{tabular}{|lcccc|}
\hline \multirow{2}{*}{ Strain } & \multicolumn{3}{c|}{ Percentage similarity (D1/D2 domain/ITS gene region) } \\
\cline { 2 - 5 } & C. rugosa ATCC 10571 & Subgroup A & Subgroup B & C. pseudorugosa XH1164 \\
\hline C. rugosa ATCC 10571 & $100 / 100$ & $99.1 / 89.2$ & $99.6 / 93.7$ & $94.6 / 81.5$ \\
C. rugosa subgroup A & $99.1 / 89.2$ & $100 / 100$ & $99.1 / 89.5$ & $94.4 / 79.5$ \\
C. rugosa subgroup B & $99.6 / 93.7$ & $99.1 / 89.5$ & $100 / 100$ & $94.6 / 81.3$ \\
C. pseudorugosa XH1164 & $94.6 / 81.5$ & $94.4 / 79.5$ & $94.6 / 81.3$ & $100 / 100$ \\
\hline
\end{tabular}

similarity with the ATCC strain. Subgroups A and B demonstrated $94.4 \%$ (27 mismatches) and $94.6 \%$ (25 mismatches) nucleotide similarity with C. pseudorugosa strain XH1164, the closest relative of C. rugosa. A search in GenBank showed that the closest match (100\%) of subgroup A was with several environmental C. rugosa isolates, and subgroup B with a few unspeciated Candida species from clinical sources (Table 2). The phylogenetic tree shown in Fig. 1(a) confirmed the clustering of the isolates with the C. rugosa ATCC 10571 strain and the close relatedness of our isolates with other C. rugosa isolates available in GenBank (Table 2).

\section{Sequence analysis of the ITS sequence data}

A total of 351 and 352 nt were determined for the ITS gene region of C. rugosa subgroup A (STC1) and B (STC4) isolates, respectively. There was no mismatch in the ITS region sequences within each subgroup. Other than the difference in sequence length, the two subgroups differed by $37 \mathrm{nt}$. The similarity among the two subgroups as determined by pairwise alignment was $90.1 \%$. Sequence analysis of the ITS region sequences showed $89.2 \%$ (29 mismatches and one deletion) and $93.7 \%$ (20 mismatches) nucleotide similarity of subgroup A and B isolates with $C$. rugosa ATCC 10571, respectively (Table 3). Subgroups A and $\mathrm{B}$ demonstrated $79.5 \%$ (71 mismatches) and $81.3 \%$ (65 mismatches) nucleotide similarity with C. pseudorugosa $\mathrm{XH1164}$, the closest relative of C. rugosa (Table 3).

Fig. 1(b) shows a dendrogram demonstrating the genetic relationship of our isolates based on ITS gene sequences of Candida reference strains and C. rugosa isolates documented in GenBank. The ITS sequences of C. rugosa subgroup A isolates showed $100 \%$ similarity only to the environmental isolates. The sequences of the subgroup $\mathrm{B}$ isolates matched (100\%) that of a C. rugosa blood isolate (strain UOA/HCPF 2) and a series of clinical isolates (from blood and pericatheter and rectal swabs) from Brazil (Table 2). The two subgroups clustered at two different branches of the ITS phylogenetic tree with a high bootstrap value (100\%).

\section{Determination of planktonic and biofilm MICs}

Table 1 shows the in vitro susceptibilities of the isolates. The MICs for ketoconazole and flucytosine ranged from 0.007 to 0.015 and from $<0.125$ to $8 \mu \mathrm{g} \mathrm{ml}^{-1}$, respectively.
High amphotericin MICs $\left(\geqslant 1 \mu \mathrm{g} \mathrm{ml}{ }^{-1}\right)$ were noted for four isolates (two from each subgroup). A blood isolate (strain STC1) from subgroup A showed dose-dependent susceptibility to fluconazole (MIC $32 \mu \mathrm{g} \mathrm{ml}^{-1}$ ). The sessile cells of all isolates displayed increased resistance to amphotericin (MIC $\geqslant 8 \mu \mathrm{g} \mathrm{ml}^{-1}$ ). Five of the six isolates had biofilm MICs of $\geqslant 128 \mu \mathrm{g} \mathrm{ml}^{-1}$ against fluconazole.

\section{DISCUSSION}

The six Malaysian isolates appeared to be identical phenotypically based on carbohydrate assimilation profiles (API 20C AUX and ID $32 \mathrm{C}$ kits), reactions on chromogenic agar (with one subgroup exhibiting more intense pigmentation), growth temperatures and enzymic characterization. Different colonial morphologies have been described for C. rugosa on CHROMagar, which include the description of rough, dry, flat colonies with a pale border resembling Candida krusei (Hospenthal et al., 2002) and small, dry colonies of a brilliant blue colour with a pale or white border indistinguishable from those of Candida albicans (Horvath et al., 2003). In this study, only one of the six isolates produced morphologically dry colonies on both potato dextrose agar and OCCA (Table 1). Based on the growth morphology and their ability to grow at $42{ }^{\circ} \mathrm{C}$, these isolates (particularly subgroup B) may be mistaken for C. albicans if the results of germ tube and other biochemical tests are not available.

Data on the epidemiology and antifungal susceptibilities of this emerging yeast pathogen are scanty. C. rugosa has been isolated most frequently from blood in outbreak situations (Behera et al., 2010; Colombo et al., 2003; Dubé et al., 1994; Singh et al., 2011) and other specimens including respiratory, skin, soft tissue and genital specimens in case reports of single cases (Minces et al., 2009; Pfaller et al., 2006). As the six isolates investigated in this study were isolated only once from our patients, this may not be sufficient for definite confirmation of their pathogenic roles, particularly for those obtained from non-sterile body sites. The origin of four of the six isolates (two from each subgroup) from blood and urine specimens in this study suggests a similarity to previous findings where C. rugosa was isolated most often from bloodstream and urinary tract infections (Minces et al., 2009; Pfaller et al., 2006). Interestingly, five of the six isolates were obtained from elderly patients ( $>60$ years old) in this study. This finding is similar to that reported by Colombo et al. (2003), where 

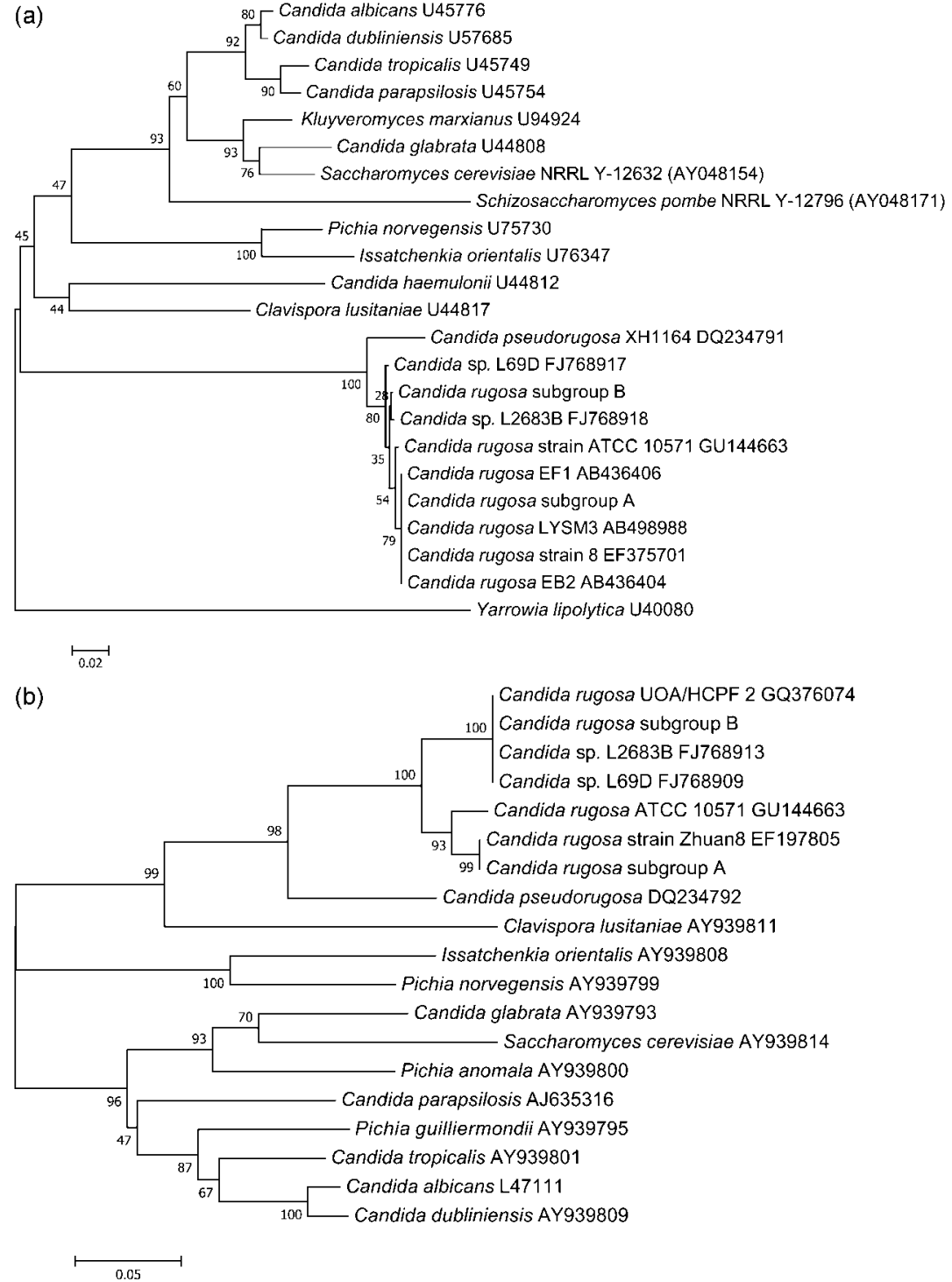

Fig. 1. Neighbour-joining tree based on the D1/ D2 domain (a) or the ITS gene region (b) depicting the relationship of $C$. rugosa subgroups $A$ and $B$ with other yeasts. The estimated confidence levels, expressed as percentages, were determined by bootstrap analysis with 1000 replications. Reference sequences were retrieved from GenBank (Table 2). the patients in five out of six C. rugosa cases in Brazil were $>65$ years old, suggesting that older individuals are at higher risk of infection with C. rugosa.

The high level $\left(\geqslant 1 \mu \mathrm{g} \mathrm{ml}^{-1}\right)$ of MICs of amphotericin for four of our isolates is a typical feature of C. rugosa (Minces et al., 2009). In addition, treatment failures with polyene agents have been reported, with isolates demonstrating in vitro susceptibility to amphotericin B (Behera et al., 2010; Colombo et al., 2003). Decreased susceptibility to fluconazole (40.5\% susceptible) was observed particularly among Asian-Pacific and Latin American isolates (Pfaller et al., 2006). Singh et al. (2011) reported that $16 \%$ of their C. rugosa isolates were resistant to fluconazole compared with $12.5 \%$ of Candida glabrata isolates and $<10 \%$ of Candida tropicalis and Candida parapsilosis isolates.

Bloodstream isolates have been reported to be the least susceptible to fluconazole (Pfaller et al., 2006). In this study, two C. rugosa blood isolates, STC1 and STC2, were considerably less susceptible to fluconazole (MICs of 32 and $4 \mu \mathrm{g} \mathrm{ml} \mathrm{m}^{-1}$, respectively) than those from other specimen types (Table 1). The susceptibility pattern of our C. rugosa isolates was therefore similar to that of $C$. glabrata and C. krusei, which are less susceptible to fluconazole and amphotericin B than most other species of Candida (Pfaller \& Diekema, 2004). Hence, accurate identification and antifungal susceptibility testing of the yeast may have a significant impact on the antifungal therapy of C. rugosa infections.

Detection of the proteinase activity from the isolates in this study suggests the potential of the isolates to cause infection, as proteinase is generally known to facilitate microbial adhesion and tissue invasion and has been identified as one of the major pathogenic determinants (Naglik et al., 2003). Identical enzymic profiles by API ZYM analysis were found for the two subgroups in this study. The enzymes detected include alkaline phosphatase, 
esterase, leucine arylamidase, acid phosphatase, esterase lipase and lipase. Of these enzymes, lipase has been postulated to facilitate proliferation of the yeast in lipidrich environments such as total parenteral nutrition (Minces et al., 2009).

Infections caused by C. rugosa are usually seen in patients within medical and surgical inpatient services and are associated with the use of central venous catheters and parenteral nutrition (Minces et al., 2009). Our finding of the ability of the isolates to form biofilms in vitro on plastic surfaces is probably correlated with previous reports of the association of C. rugosa with catheter-related infections and resistance against therapy (Colombo et al., 2003; Minces et al., 2009; Rosas et al., 2004). The increased resistance to amphotericin (MIC $\geqslant 8 \mu \mathrm{g} \mathrm{ml}^{-1}$ ) and fluconazole (MIC $\geqslant 128 \mu \mathrm{g} \mathrm{ml}^{-1}$ ) of the sessile cells compared with planktonic cells is similar to the observation for other Candida species at the biofilm stage (Ramage et al., 2001).

The organisms identified in this study are among a number of novel yeast species in clinical specimens that cannot be distinguished phenotypically from an existing species and rely on a molecular approach for identification (Ciardo et al., 2006). The D1/D2 domain and ITS sequences of our isolates do not match any known yeast species in GenBank or the CBS yeast sequence database (http://www.cbs.knaw. $\mathrm{nl} /$ collections/). Kurtzman \& Robnett (1997) predicted that yeast strains showing $>1 \%$ substitutions in the $\sim 600 \mathrm{nt}$ D1/D2 domain are likely to be different species and that strains with $0-3 \mathrm{nt}$ mismatches are either conspecific or sister species. Hence, based on this correlation, subgroup B (with two mismatches compared with the type strain) is most probably conspecific with C. rugosa.

Although subgroup A had only $0.9 \%$ substitutions in its D1/D2 domain (four mismatches) compared with the type strain, the greater ITS sequence variability $(10.8 \%)$ of this subgroup supports the suggestion that the yeast be recognized as a distinct species, as the percentage intraspecies similarity reported previously for C. rugosa is $94.5-$ $100 \%$ (Ciardo et al., 2006). However, the predicted conspecificity and proposal of a novel Candida species will require more detailed taxonomic reassessment using DNADNA hybridization assays (Kurtzman \& Robnett, 1997) or other more sophisticated molecular approaches.

The source of $C$. rugosa is unknown. The yeast is frequently isolated from the milk of mastitic cows (Crawshaw et al., 2005) and its lipase has been used for food production (Benjamin \& Pandey, 1998). Hence, contaminated milk or food products have been speculated as a source of infection (Barton, 2011). Sequence comparison in this study demonstrated the association of subgroup A isolates (only reported from environmental sources so far) with human infections for the first time, following identification of the isolates from different clinical specimens (blood, urine and bronchoalveolar lavage) in this study. Isolation of the subgroup B isolates from various clinical specimens in this and other studies (as documented in GenBank) is an indication of the emergence of this yeast as a human pathogen in various geographical regions.

Over the past few years, several new and emerging yeast pathogens misidentified using conventional chemotaxonomic criteria have been reported by sequence analysis of the D1/D2 domain and ITS sequences. Molecular techniques have assisted in the identification of C. pseudorugosa (Li et al., 2006) and Candida pararugosa (Ciardo et al., 2006), which were misidentified previously as C. rugosa using phenotypic methods. In this and the abovementioned studies, the ITS region has been shown to be a valuable region for differentiation of closely related subgroups of C. rugosa (tentatively referred to as the $C$. rugosa complex). Further clarification of the complex taxonomy of C. rugosa will assist in our understanding of the epidemiology and clinical significance of this yeast.

\section{ACKNOWLEDGEMENTS}

This study was supported by research grants from the University of Malaya (nos RG090-09HTM and FS240/2008B). We thank Dr Marizeth Groenewald, Curator Yeast Division, CBS-KNAW Fungal Biodiversity Centre, Utrecht, The Netherlands, for assistance in searching for similar sequences in the CBS database. We thank Professor K. P. Ng and Ms I. A. Zainal Abidin for their help and contributions to this study.

\section{REFERENCES}

Barton, R. C. (2011). Candida rugosa: a new fungal pathogen emerging, but from where? J Med Microbiol 60, 265-266.

Behera, B., Singh, R. I., Xess, I., Mathur, P., Hasan, F. \& Misra, M. C. (2010). Candida rugosa: a possible emerging cause of candidaemia in trauma patients. Infection 38, 387-393.

Benjamin, S. \& Pandey, A. (1998). Candida rugosa lipases: molecular biology and versatility in biotechnology. Yeast 14, 1069-1087.

Ciardo, D. E., Schär, G., Böttger, E. C., Altwegg, M. \& Bosshard, P. P. (2006). Internal transcribed spacer sequencing versus biochemical profiling for identification of medically important yeasts. J Clin Microbiol 44, 77-84.

CLSI (2008). Reference Method for Broth Dilution Antifungal Susceptibility Testing of Yeasts, 3rd edn; Approved Standard. M27A3. Wayne, PA: Clinical and Laboratory Standards Institute.

Colombo, A. L., Melo, A. S. A., Crespo Rosas, R. F., Salomão, R., Briones, M., Hollis, R. J., Messer, S. A. \& Pfaller, M. A. (2003). Outbreak of Candida rugosa candidemia: an emerging pathogen that may be refractory to amphotericin B therapy. Diagn Microbiol Infect Dis 46, 253-257.

Crawshaw, W. M., MacDonald, N. R. \& Duncan, G. (2005). Outbreak of Candida rugosa mastitis in a dairy herd after intramammary antibiotic treatment. Vet Rec 156, 812-813.

Dubé, M. P., Heseltine, P. N. R., Rinaldi, M. G., Evans, S. \& Zawacki, B. (1994). Fungemia and colonization with nystatin-resistant Candida rugosa in a burn unit. Clin Infect Dis 18, 77-82.

Horvath, L. L., Hospenthal, D. R., Murray, C. K. \& Dooley, D. P. (2003). Direct isolation of Candida spp. from blood cultures on the chromogenic medium CHROMagar Candida. J Clin Microbiol 41, 2629-2632. 
Hospenthal, D. R., Murray, C. K., Beckius, M. L., Green, J. A. \& Dooley, D. P. (2002). Persistence of pigment production by yeast isolates grown on CHROMagar Candida medium. J Clin Microbiol 40, $4768-4770$.

Jin, Y., Yip, H. K., Samaranayake, Y. H., Yau, J. Y. \& Samaranayake, L. P. (2003). Biofilm-forming ability of Candida albicans is unlikely to contribute to high levels of oral yeast carriage in cases of human immunodeficiency virus infection. J Clin Microbiol 41, 2961-2967.

Kumar, S., Tamura, K. \& Nei, M. (2004). MEGA3: integrated software for Molecular Evolutionary Genetics Analysis and sequence alignment. Brief Bioinform 5, 150-163.

Kurtzman, C. P. \& Robnett, C. J. (1997). Identification of clinically important ascomycetous yeasts based on nucleotide divergence in the $5^{\prime}$ end of the large-subunit (26S) ribosomal DNA gene. J Clin Microbiol 35, 1216-1223.

Li, J., Xu, Y. C. \& Bai, F. Y. (2006). Candida pseudorugosa sp. nov., a novel yeast species from sputum. J Clin Microbiol 44, 4486-4490.

Makimura, K., Murayama, S. Y. \& Yamaguchi, H. (1994). Detection of a wide range of medically important fungi by the polymerase chain reaction. J Med Microbiol 40, 358-364.

Melo, A. S., Colombo, A. L. \& Arthington-Skaggs, B. A. (2007). Paradoxical growth effect of caspofungin observed on biofilms and planktonic cells of five different Candida species. Antimicrob Agents Chemother 51, 3081-3088.

Minces, L. R., Ho, K. S., Veldkamp, P. J. \& Clancy, C. J. (2009). Candida rugosa: a distinctive emerging cause of candidaemia. A case report and review of the literature. Scand J Infect Dis 41, 892-897.

Naglik, J. R., Challacombe, S. J. \& Hube, B. (2003). Candida albicans secreted aspartyl proteinases in virulence and pathogenesis. Microbiol Mol Biol Rev 67, 400-428.

Pfaller, M. A. \& Diekema, D. J. (2004). Rare and emerging opportunistic fungal pathogens: concern for resistance beyond
Candida albicans and Aspergillus fumigatus. J Clin Microbiol 42, 4419-4431.

Pfaller, M. A., Diekema, D. J., Colombo, A. L., Kibbler, C., Ng, K. P., Gibbs, D. L., Newell, V. A. \& Global Antifungal Surveillance Group (2006). Candida rugosa, an emerging fungal pathogen with resistance to azoles: geographic and temporal trends from the ARTEMIS DISK Antifungal Surveillance Program. J Clin Microbiol 44, 3578-3582.

Ramage, G., Vande Walle, K., Wickes, B. L. \& López-Ribot, J. L. (2001). Standardized method for in vitro antifungal susceptibility testing of Candida albicans biofilms. Antimicrob Agents Chemother 45, 2475-2479.

Redkar, R. J., Dubé, M. P., McCleskey, F. K., Rinaldi, M. G. \& Del Vecchio, V. G. (1996). DNA fingerprinting of Candida rugosa via repetitive sequence-based PCR. J Clin Microbiol 34, 1677-1681.

Rosas, R., Nucci, M., Castelo, A. \& Colombo, A. L. (2004). Predictive value of Candida spp. colonization in the diagnosis of candidemia in intensive care unit patients. In Program and Abstracts of the 44th Interscience Conference on Antimicrobial Agents and Chemotherapy, p. 410, abstract M-269. Washington, DC: American Society for Microbiology.

Ruma-Haynes, P. A., Brownlee, A. G. \& Sorrell, T. C. (2000). A rapid method for detecting extracellular proteinase activity in Cryptococcus neoformans and a survey of 63 isolates. J Med Microbiol 49, 733-737.

Singh, R. I., Xess, I., Mathur, P., Behera, B., Gupta, B. \& Misra, M. C. (2011). Epidemiology of candidaemia in critically ill trauma patients: experiences of a level I trauma centre in North India. J Med Microbiol 60, 342-348.

White, T. J., Bruns, T. D., Lee, S. B. \& Taylor, J. W. (1990). Amplification and direct sequencing of fungal ribosomal DNA for phylogenetics. In PCR Protocols: a Guide to the Methods and Applications, pp. 315-322. Edited by M. A. Innis, D. H. Gelfand, J. J. Sninsky \& T. J. White. New York: Academic Press. 